\title{
Representaciones sociales docentes y neurociencias educativas: posibles vínculos para el caso argentino en torno al aula intercultural
}

\author{
Teachers' social representations and educational neurosciences: \\ possible links for the Argentine case around the intercultural classroom \\ Paula Luciana Buratovich \\ Instituto de Investigaciones Gino Germani \\ Facultad de Ciencias Sociales \\ Universidad de Buenos Aires \\ paulaburatovich@hotmail.com \\ Anahí González \\ Instituto de Investigaciones Gino Germani \\ Facultad de Ciencias Sociales \\ Universidad de Buenos Aires \\ anahipgonzalez@gmail.com \\ Romina Tavernelli \\ Instituto de Investigaciones Gino Germani \\ Facultad de Ciencias Sociales \\ Universidad de Buenos Aires \\ rptavernelli@gmail.com
}

\section{Resumen:}

El propósito del siguiente artículo es presentar una investigación en curso que, desde una perspectiva sociológica centrada en el análisis de representaciones sociales, busca explorar de qué manera se articulan los discursos provenientes de las neurociencias educativas con los modos en que se gestiona la interculturalidad en la escuela. La indagación se estructura en torno a la hipótesis de que las representaciones sociales que miembros del campo educativo construyen acerca de la presencia y el desempeño escolar de niños y niñas migrantes se ven influenciadas por el auge de los discursos normalizadores de las neurociencias educacionales que se instauran como nuevas modalidades de gestión, disciplinamiento y control social de la diversidad (migratoria) en la institución escolar, coadyuvando en la reproducción de procesos de exclusión. En consecuencia, el objetivo general de dicha investigación apunta a indagar el modo en que los discursos surgidos de las investigaciones en el campo de las neurociencias educacionales, desarrolladas desde 1990 a la actualidad, inciden en las representaciones sociales que miembros de escuelas primarias y secundarias del área metropolitana de Buenos Aires construyen en torno al desempeño escolar de poblaciones en situación de vulnerabilidad, tales como niños, niñas y adolescentes de origen migrante. La intención es focalizar en la presencia de discursos tendientes a legitimar acciones pedagógicas estigmatizantes sobre dichas poblaciones.

Paula Luciana Buratovich · Anahí González · Romina Tavernelli Representaciones sociales docentes y neurociencias educativas: posibles vínculos para el caso argentino en torno al aula intercultural. Autoctonía. Revista de Ciencias Sociales e Historia, Vol. III, N² 2, Julio-Diciembre 2019, 185-200 ISSN 0719-8213

DOI: http://doi.org/10.23854/autoc.v3i2.121 
Palabras clave: Neurociencias educativas, interculturalidad, representaciones sociales, diversidad migratoria

\begin{abstract}
:
The purpose of this article is to introduce a research project in progress that analyzes, from a sociological perspective based in social representations analysis, how discourses on educational neuroscience are articulated with the ways that intercultural relations within the school space are managed. The hypothesis that guides the research suggests that the social representations that members of the educational field construct about the presence and the school performance of children of migrant origin, are influenced by the relevance of the normalizing discourses of the educational neurosciences that are established as new modalities of management, discipline and social control of diversity (migratory) in the school, contributing to the reproduction of exclusion processes. The general objective of the project is to investigate how discourses coming from researches in the field of educational neuroscience, developed from 1990 to the present, influence the social representations of members of primary and secondary schools in the metropolitan area of Buenos Aires on migratory diversity. The purpose is to focus on the presence of discourses tending to legitimize stigmatizing pedagogical actions on migrant otherness.
\end{abstract}

Keywords: Educational neurosciences, interculturality, social representations, migratory diversity

Recibido: 29 de Junio de 2019 - Aceptado: 13 de Agosto de 2019

\section{Introducción}

Históricamente las instituciones educativas han sido caja de resonancia de diversos desarrollos del conocimiento científico. Desde hace algunos años, asistimos a una diseminación y masificación de ofertas de formación, charlas, publicaciones - todos recursos "pensados" y "adaptados" para los docentes y su práctica áulica- que difunden contenidos de la neurociencia educacional.
"No es que las neurociencias vayan a responder a todas las preguntas que surgen en las prácticas educativas, pero sus aportes son relevantes para repensar los procesos de enseñanza y aprendizaje y cómo mejorarlos. Es posible que varios principios de este enfoque vayan en línea con lo que muchos docentes intuyen y ya hacen en su día a día. En estos casos, las neurociencias podrán confirmar y apoyar científicamente esas prácticas..." (Ministerio de Educación de la Nación, s/f.: 2) 
Esta frase inaugura la colección "Aprender con el cerebro en mente", material editado en Argentina por el Ministerio de Educación de la Nación y el Instituto de Neurociencias y Educación (INE) de la Fundación INECOํ․ Dicho material no responde a una iniciativa aislada, sino que se trata de un ejemplo más del auge que las neurociencias -y en particular, la neurociencia educativa (o neuroeducación)- están experimentando tanto en Argentina como en el mundo.

La neurociencia educativa, considerada una subdisciplina de la ciencia básica de la neurociencia cognitiva, se ocupa de estudiar, en términos de actividad neural, cómo se llevan a cabo los procesos cognitivos y de enseñanza y aprendizaje. Su proliferación ha motivado nuestro interés por explorar de qué manera se articulan los discursos provenientes de esta disciplina con los modos en que se gestiona la interculturalidad en la institución educativa. Luego de un largo recorrido de investigación en torno a la temática de la diversidad migratoria en la escuela, nos proponemos presentar en este artículo los principales interrogantes e hipótesis que dieron lugar a un proyecto de investigación en curso que, desde una perspectiva sociológica y de carácter exploratorio, pretende indagar el vínculo entre las representaciones sociales de miembros del campo educativo acerca de la diversidad migratoria y las investigaciones fundadas en la neurociencia educacional.

El artículo se estructura del siguiente modo. En primer lugar exponemos los elementos princi- pales del proyecto de investigación del cual se desprenden las ideas a desarrollar en el texto. Así, especificamos la hipótesis y objetivos que guían la indagación. En segundo término, presentamos el debate de las principales corrientes sobre las neurociencias educativas entendiéndolas como entramados discursivos generadores de sentido sobre el proceso de enseñanza-aprendizaje y los sujetos que participan en él. Seguidamente, desarrollamos algunas consideraciones en torno al vínculo entre el Estado Nación, la escuela y la interculturalidad, planteando algunas reflexiones en relación con los discursos de las neurociencias educativas y las eventuales consecuencias de su impacto en la gestión de la diversidad migratoria. Finalmente, a modo de cierre, proponemos algunas consideraciones finales que orientan nuestras líneas de investigación a futuro.

\section{Hacia un análisis de los vínculos entre neu- rociencias educativas, interculturalidad y dis- criminación. El inicio de una investigación}

En su complejo proceso de formación y consolidación, el Estado Nación encomendó a la institución educativa, en tanto agente de socialización de gran alcance, la ardua tarea de homogeneizar poblaciones diversas. Teniendo en cuenta que la sociedad argentina ha estado atravesada por las migraciones, la escuela pública, a lo largo de su historia, fue concebida como un espacio capaz de amalgamar la diversidad y colaborar en la formación del llamado "ser nacional". (Bertoni, 2007) 
Actualmente, la neurociencia educativa goza de una creciente presencia en la agenda pública, tanto en los discursos de personalidades políticas como de medios de comunicación. Esta disciplina promete ofrecer, mediante el establecimiento de un "puente" entre los hallazgos científicos y el aula, herramientas que permitirían optimizar los procesos de enseñanza y aprendizaje a partir de las exploraciones acerca del funcionamiento neural.

Con el propósito de vincular dicha histórica función atribuida a la escuela -y el modo en que en ella se ha gestionado la diversidad- con el auge que los discursos provenientes de la neurociencia están experimentando en el mundo educativo, proponemos un abordaje que parte de una perspectiva sociológica centrada en el estudio de las representaciones sociales de la sociedad receptora en torno a la migración; y entrecruza los análisis sobre interculturalidad, discriminación y exclusión en el sistema educativo con la neurociencia educativa en tanto disciplina capaz de brindar insumos para elaborar dispositivos y técnicas de normalización y disciplinamiento.

Siguiendo a Jodelet, asumimos las representaciones sociales como "modalidades de pensamiento práctico orientados hacia la comunicación, la comprensión y el dominio del entorno social, material e ideal" (1986: 472). En tal sentido, son una manera de pensar e interpretar nuestra realidad cotidiana y por ello una forma de conocimiento social que, en una imagen cosificante, condensan historia, relaciones sociales y prejuicios (Jodelet, 1986).

En nuestro proyecto de investigación, partimos de la hipótesis que plantea que los discursos normalizadores de las neurociencias educacionales inciden sobre las representaciones sociales que los miembros del campo educativo construyen acerca de la presencia y el desempeño escolar de niños, niñas y adolescentes de origen migrante, instaurándose como nuevas modalidades de gestión, disciplinamiento y control social de dicha diversidad coadyuvando en la reproducción de procesos más generales de exclusión social. En ese sentido, nos hemos propuesto como objetivo general de la investigación indagar el modo en que los discursos surgidos de las investigaciones en el campo de las neurociencias educacionales, desarrolladas desde 1990 la actualidad, inciden en las representaciones sociales que miembros de escuelas primarias y secundarias del área metropolitana de Buenos Aires construyen en torno a la presencia y desempeño escolar de poblaciones en situación de vulnerabilidad, tales como niños, niñas y adolescentes de origen migrante, focalizando en la presencia de discursos tendientes a legitimar acciones pedagógicas estigmatizantes respecto de tales poblaciones.

Consecuentemente, en nuestra investigación procuramos explorar las representaciones sociales de docentes, diseñadores y gestores de políticas públicas educativas que refieren al rol social de la escuela, rastreando posibles funciones vinculadas a la normalización y/o control de las poblaciones migrantes y a la producción de un 
sujeto construido en términos de éxito/fracaso; e identificar en dichas representaciones aquellos elementos discursivos que complementan y/o ponen en tensión los postulados de la meritocracia y la neurociencia educacional. Asimismo, exploraremos las formas en que los discursos de la neurociencia educacional tienden, a partir de explicaciones biologicistas, a la normalización de las poblaciones migrantes; y rastrearemos los llamados "neuromitos", entendidos como "un concepto erróneo generado por una mala lectura o interpretación de hechos científicamente establecidos por las investigaciones sobre el cerebro" (OCDE, 2002), en las representaciones sociales de los docentes, y su vinculación con la construcción de la otredad migrante presente en el aula.

\section{Neurociencias y educación: principales debates}

Los primeros antecedentes sobre neurociencia educacional, es decir en los que se produjeron intentos por vincular neurociencia y educación, pueden rastrearse hacia mediados del siglo pasado. Sin embargo, será promediando la década de 1990 cuando surgirán los debates contemporáneos, en los términos en que hoy se están dando, sobre la neurociencia educacional. Son señalados por la literatura del tema tres momentos importantes, casi fundantes del vínculo entre ambas disciplinas. El primero de ellos es un encuentro organizado por la Comisión de Educación de los Estados y la Fundación Dana en 1996, en el que especialistas en la materia debatieron sobre los avances de la neurociencia cognitiva y la psicología cognitiva vinculada a la educación. En un documento producido por los participantes de ese encuentro se realizaban diversas recomendaciones comunicacionales para la difusión de conceptos centrales de la neurociencia (aprendizaje, memoria, períodos críticos y niveles de organización), así como sugerencias con relación al diseño de políticas educativas a futuro.

Los otros dos sucesos fueron la publicación de dos trabajos inaugurales en este campo: Education and the Brain: A bridge too far, de Bruer (1997) y The Educational relevance of research in cognitive neuroscience, de Byrnes y Fox, quienes lo publicaron en 1998.

En su artículo Byrnes y Fox (1998), consideran los beneficios de incorporar los hallazgos de la neurociencia cognitiva en el campo de la psicología educativa. Plantean argumentos en oposición a la idea de que se puede ignorar el cerebro cuando se postula un modelo de aprendizaje o motivación del estudiante, detallan las limitaciones en los métodos utilizados para revelar las relaciones cerebro-cognición, se describen las propiedades del cerebro y el desarrollo del cerebro, y resumen la investigación neurocientífica cognitiva sobre atención, memoria, lectura y matemáticas. Además, sugieren áreas de investigación futuras en neurociencia cognitiva.

En Education and the Brain: A bridge too far (1997), texto central en la literatura sobre neurociencias educativas, Bruer afirmaba que el puente entre ambas disciplinas era aún "muy le- 
jano", ya que muchos de los aportes de las neurociencias se realizaban con base en experimentos en animales y difícilmente podían extrapolarse a los seres humanos. Consecuentemente, el autor enfatizaba en que debía primar la cautela al realizar afirmaciones con relación a los aportes de la neuroeducación. El principal objetivo de dicho trabajo fue prevenir que las concepciones "neuro" erróneas influyeran y permearan los programas educativos y diseños curriculares, principalmente en lo referente a la sinaptogénesis, a la existencia de períodos críticos en el desarrollo, y a los efectos en el cerebro del enriquecimiento del ambiente. Casi veinte años después de aquella afirmación, Bruer (2016) sostiene que la neurociencia educativa no es todavía una ciencia aplicada, sino una ciencia básica que puede contribuir a la investigación básica en educación, pero no necesariamente en forma directa a la enseñanza en el aula.

Seguidamente a aquellos trabajos de la década de 1990, se multiplicarán las publicaciones acerca de la temática (Lipina, 2016). Siendo esquemáticos, podemos decir, a modo de un primer resumen, que -entre los debates en auge- existen básicamente tres posturas: aquellas que plantean el rechazo absoluto de lo propuesto por las neurociencias educacionales, dada la biologización y medicalización que su aplicación en el diseño de políticas públicas sobre educación pudieran significar (Merlin, 2017; Carli, 2017); en segundo lugar, las que sostienen que las incipientes investigaciones no pueden ser concluyentes, advirtiendo a la vez acerca de las lecturas sim- plistas y el surgimiento de "neuromitos" (Terigi, 2016; Pallarés Domínguez, 2016) afirmando que las neurociencias, por el momento, deben ser pensadas tan solo en su aspecto de ciencia básica (Lipina, 2016) y, por último, quienes realizan una defensa férrea sobre los aportes que las mismas tendrían en el ámbito de la educación y el aprendizaje entendiendo que aquel puente entre neurociencia y educación ya puede cruzarse (Manes, 2013; Raspall, 2017).

Resulta esclarecedor el aporte de Terigi (2016: 51) quien en su artículo "sobre aprendizaje escolar y neurociencias", sostiene que este auge de la neurociencia educacional forma parte de un problema de mayor envergadura consistente en "el desplazamiento de las formulaciones pedagógicas por formulaciones de otras disciplinas" y en el que la divulgación científica sobre la temática cumple un rol central en la difusión de lo que la perspectiva crítica llama "neuromitos", entendidos como "falsas ideas, creencias, interpretaciones o extrapolaciones que han trascendido a la opinión pública a pesar de haber sido desterradas o invalidadas por la neurociencia..." sobre todo a partir de su difusión en medios de comunicación y que por ello cuesta mucho desarraigar. (Pallarés Domínguez, 2016: 944).

Entre los "neuromitos" más extendidos pueden mencionarse las ideas que refieren a que "solo usamos el $10 \%$ del cerebro", que "los problemas de aprendizaje asociados con diferencias en el desarrollo de funciones cerebrales no pueden ser remediados por la educación", que "hay pe- 
ríodos críticos durante la niñez, luego de los cuales algunos aprendizajes ya no pueden lograrse"; o la idea de que "la utilización preferente de un hemisferio del cerebro condiciona el aprendizaje"; o aquellas que remiten a "los estilos preferentes de aprendizaje: visual, auditiva, kinestésica”; entre otros. (Pallarés Domínguez, 2016). Sobre los "neuromitos" Dekker, Lee, Howard Jones y Jolles (2012) presentaron una investigación en la que encuestaron a 242 profesores de escuelas primarias y secundarias ubicadas en el Reino Unido y los Países Bajos interesados en las neurociencias aplicadas a la educación y encontraron que el $49 \%$ de los neuromitos fueron corroborados. Según los investigadores, estos hallazgos sugieren que los docentes entusiastas de la aplicación de las neurociencias a la educación no distinguen con facilidad entre pseudociencia y hechos científicos.

En sintonía con ello, los puntos de mayor debate acerca de las neurociencias se centran en las consecuencias que podría acarrear el hecho de que sus postulados fueran llevados a la "práctica educativa”.

Al respecto, Castorina (2016), sin pretensión de evaluar sus hallazgos, examina las cuestiones filosóficas y de sentido que la neurociencia educativa supone, recuperando los debates acerca de su aplicabilidad en el aula planteando que la actividad del cerebro y su estudio es condición necesaria pero no suficiente para aprehender los procesos de aprendizaje, analizando además la cuestión de una perspectiva interdisciplinar.
Bowers (2016) y De Vos (2016) simplemente afirman que los aportes que las neurociencias dicen otorgar a la práctica docente son, en su mayoría, autoevidentes (tales como las dificultades de aprendizaje relacionadas con entornos estresantes, malos hábitos de sueño y falta de ejercicio) y que a su vez la supuesta ayuda que vendrían a traer podría generar más problemas que soluciones a los educadores.

Algunos autores enfatizan la importancia de trabajar interdisciplinariamente con otros campos antes de descartar la neurociencia educativa, sobre todo con aquellos que tienen como punto de partida la mente, en lugar del cerebro. (Marcus, 2013)

En Latinoamérica, en general, y en Argentina, en particular, se evidencia una creciente propagación de discursos referentes a la neurociencia, la educación y su aplicación en la práctica docente. (Ferreres y Abusamra, 2016; Battro, 2011; Battro y Cardinali, 1996; Manes, 2013.)

Un informe de la OCDE (2007:32) que analiza de modo positivo los aportes de las neurociencias en el área educativa plantea que

“...de ninguna manera la neurociencia es la panacea -y que comenzará una revolución en educación, sobre todo no inmediatamente-. Los líderes del proyecto han advertido una y otra vez que la "neurociencia por sí misma es poco probable que resuelva todos, si es que alguno de los temas educacionales". Las respuestas a muchas de las 
interrogantes educacionales pueden encontrarse en otra parte, ya sea dentro de la educación misma o en otras disciplinas referentes a las ciencias sociales, o sin duda, en la filosofía."

Aun así, crecen las iniciativas -tanto estatales como privadas- que aseguran que las neurociencias educativas tienen mucho para decirnos, ya sea en tanto herramientas e insumos teóricos para el desarrollo de políticas públicas sobre educación como así también en cuanto instrumentos analíticos para el diseño de estrategias didácticas de aplicación directa en el aula.

\section{Estado nación, escuela y diversidad migra- toria}

La institución escolar y la diversidad han sido objeto de análisis desde diversas disciplinas. Entre ellas, sin duda, los aportes fundamentales al debate acerca de la interculturalidad provienen de las ciencias de la educación, la antropología y la sociología. Desde la antropología, un libro que ya se ha convertido en un clásico sobre el tema y que debe mencionarse es "De eso no se habla... los usos de la diversidad sociocultural en la escuela" compilado por Neufeld y Thisted (2001) en el que se resumen los resultados de pesquisa acerca de procesos vinculados con la diversidad en las escuelas, abordando temas tales como la discriminación, el prejuicio, los estereotipos, la racialización, etc. En dicho libro, Sinisi (2001: 200) entiende por "espacios escolares "multiculturales", precisamente a la intersección de las múltiples variables que abrevan en las escuelas como ser, el barrio en el que está inserta, la situación social, la cultura, la nacionalidad, etc. de los sujetos que en ellas encontramos (docentes, alumnos, padres y otros integrantes de la comunidad escolar), pero sin olvidar que estos espacios se constituyen y cobran significatividad en tanto son producidos en contextos históricos determinados y en determinadas relaciones de poder...". Otros trabajos que también toman en cuenta a la escuela como espacio para el estudio de la diversidad migratoria que pueden mencionarse son Duschatzky (1996), Duschatzky y Skliar (2000), Gagliano (1991), Jordán (1994), Juliano (1993), Mccarthy (1994), Finocchio (2009), Jordán Sierra (2003), Cebolla Boado (2007), Diez y Novaro (2011), Diez, Novaro y Martínez (2017), entre muchos otros. Cada uno de estos trabajos aborda las temáticas de la multiculturalidad, la interculturalidad y la diversidad como fenómenos que implican que, al tomarse conciencia de la presencia de identidades diferentes, se plantean nuevos desafíos a los modos de pensar y afrontar la existencia subjetiva. En esta coyuntura comienzan a circular discursos que mencionan y proponen prácticas de "integración”, “respeto a la diferencia”, “aceptación de la diversidad", etc., así como también problematizan las perspectivas asimilacionistas.

Como resultado de investigaciones previas en torno a la cuestión de la interculturalidad y las representaciones sociales que se construyen y reproducen desde la sociedad receptora sobre la migración, entendemos que hay dos dimensiones del fenómeno intercultural en Argentina 
que le otorgan un perfil propio. En primer término, se evidencian obstáculos materiales y simbólicos-arraigados en las etapas de constitución de la "Nación argentina"- para la producción de una idea de nación plural, intercultural y étnicamente diversa. En segundo lugar, instituciones como la escuela continúan reproduciendo imaginarios vinculados a la homogeneización de la sociedad, sin cuestionar profundamente los modos en que se configura el fenómeno intercultural en Argentina.

En este sentido, Bauman (2003) plantea que las sociedades receptoras de migrantes tienden a elaborar estrategias que tienen como objetivo construir un discurso y un hacer único que licue la diversidad, destinando al "otro" a un lugar que evite poner en riesgo el orden, controlándolo. Entendemos que ese es el lugar del migrante externo, principalmente sudamericano, en nuestro país.

En ese sentido, asumimos que el Estado-nación -y a través de él, los distintos gobiernos- gestiona la presencia de la diversidad migratoria no sólo a través de su corpus jurídico y normativo sino, más ampliamente, desde el accionar cotidiano de sus instituciones. Asimismo, entendemos que la sociedad receptora condensa sus representaciones sociales en las instituciones que ella erige, "hablando" a través de ellas, pues "construimos las instituciones, al comprimir las ideas de todos para darles una forma común de modo que se pueda demostrar su corrección en virtud del peso abrumador del número de actos inde- pendientes de asentimiento" (Douglas, 1996: 135). En cuanto a la relación entre instituciones y representaciones sociales, coincidimos en que "las instituciones guían de manera sistemática a la memoria individual y encauzan nuestra percepción hacia formas que resultan compatibles con las relaciones que ellas autorizan" (Douglas, 1996: 137).

La escuela, tanto por el universo sobre el que actúa como por el tiempo que ella está presente en la vida de los sujetos, resulta una institución que no puede soslayarse al momento de estudiar la gestión de la diversidad migratoria por parte del Estado. En la actualidad, resulta innegable la histórica función integradora y a la vez normalizadora, que ha tenido (y tiene) en nuestro país, teniendo en cuenta que, desde sus orígenes, el Estado nación argentino ha estado atravesado por movimientos migratorios, lo cual complejizó el proceso de consolidación de una única identidad nacional. La escuela tuvo un rol protagónico, atribuido por el hecho mismo de ser un espacio privilegiado de encuentro de niños y niñas tanto de origen nacional como extranjero.

En definitiva, la labor de la institución educativa ha estado también orientada a "resolver" la diversidad en pos de una homogénea y única identidad nacional.

Adicionalmente, resulta interesante estudiar la institución escolar dado el poder performativo de las acciones cotidianas que allí se desarrollan, así como los discursos de los distintos acto- 
res que en ella se encuentran, pues dan cuenta de cuán conflictiva puede ser dicha convivencia y de qué modo la escuela puede configurarse como un lugar en el que también se reproducen desigualdades. Sin embargo, cabe señalar que el rol social que se le asigna a la escuela es muy distinto. La imagen acerca de que la educación por sí misma contribuye a la movilidad social ascendente y que en ella se trabaja para lograr la "integración de los migrantes" se encuentra sumamente instalada en la sociedad, por lo que detectar acciones que la contradigan, entraña un desafío.

Como resultado de otras investigaciones podemos afirmar que entre los docentes aún perviven aquellas representaciones sociales que vinculan a la escuela, no sólo con la igualdad de oportunidades, sino con la percepción acerca de que dicha igualdad encuentra sus antecedentes y fundamentos en la meritocracia y el "éxito individual".

Por su parte, la emergencia y progresiva consolidación de un modelo basado en la igualdad de oportunidades, halla su base argumental en la meritocracia entendida como un sistema objetivo de distribución de los provechos y beneficios que la sociedad pondría al alcance de todos, indistintamente. En tal sentido, las desigualdades dejarían de ser injustas, pues todos los individuos aparecen como poseedores de las mismas oportunidades que les permitirían superar aquellas. Tal como afirma Dubet (2011: 62 ), "dado que las oportunidades conciernen a los individuos, éstos deben ser activos y movilizarse para merecerlas”. Se afirma así la cultura del empowerment, un modelo que tiene al individuo como protagonista de su propio destino, operando un pasaje de políticas universales a políticas centradas en "públicos, riesgos y oportunidades específicas” (Dubet, 2011: 62).

Es evidente que tal concepción conlleva a la transformación de las representaciones de la vida social en el más amplio de los sentidos.

En este escenario, entendemos que se erige una escuela que consagra la meritocracia, y en la que subyace la idea de la supervivencia del más apto consolidando un modelo social basado en la suma de contratos individuales.

Por su parte, el discurso de las neurociencias comienza a colarse en los debates científicos y políticos en nuestro país, proponiendo nuevas alianzas entre el conocimiento de laboratorio y las políticas públicas. En este contexto, la tentación de vincular el discurso de las neurociencias al mejoramiento o cambio en los procesos de enseñanza aprendizaje, va en aumento. A partir del avance de nuevas técnicas de exploración neural, se ha podido aumentar el conocimiento con relación a determinados procesos vinculados con la enseñanza y el aprendizaje, tales como la atención, la motivación, las emociones, el lenguaje, la memoria, entre otros (Terigi, 2016). En este sentido, y como se mencionó precedentemente, si bien la investigación contemporánea -escapando a una visión fijista acerca de las ba- 
ses biológicas de la conducta y el aprendizaje-, incorpora cuestiones que relacionan el sistema nervioso con las experiencias de los sujetos, resulta innegable la imposibilidad, aún, de aplicación y traslado directo de los resultados de las investigaciones de la neurociencia al estudio del aprendizaje escolar.

Sin dejar de lado la importancia del trabajo interdisciplinario, sería un grave error dar ese salto en la actualidad sin correr el riesgo de reproducir concepciones biologicistas en el ámbito de lo social. En este sentido, afirma Terigi (2016), "una cosa es aceptar que 'todo proceso cognitivo es un proceso material, que se da como un conjunto de funciones sostenidas por estructuras neuronales que permiten el desarrollo de dicho proceso' [Maureira, 2010: 451. Citado en: (Terigi, 2016: 54)] y otra muy distinta es pretender reducir las caracterizaciones del aprendizaje escolar a lo que surge de la investigación sobre el desarrollo de funciones cerebrales."

Sin embargo, a partir de algunas recientes decisiones que desde el Estado argentino se han llevado adelante, pareciera que tal salto es posible y, más aún, que se aspira a generar políticas públicas educativas de gran alcance. Así, el riesgo que estas decisiones pueden acarrear entendemos tendría un significativo impacto, en especial hacia las poblaciones vulnerables y, en particular para nuestro caso, sobre las poblaciones de origen migrante.

Frente a este escenario, cabe preguntarse cuál

sería el efecto que podría tener en la cotidianeidad del aula intercultural la adopción de la moda de agregar a toda investigación el prefijo "neuro" y trasladar la incorporación de nociones emergentes de investigaciones aún en curso que, fundamentalmente, pertenecen a otro campo disciplinar. Más aún, podría pensarse que las representaciones en torno a tales investigaciones, conducirían a la reproducción de lo que se ha dado en llamar "neuromitos", es decir, interpretaciones erróneas acerca de cómo trabaja el cerebro, que trasladarían-como todo prejuicio- la reproducción de estereotipos y, por ende, de desigualdades más estructurales.

En definitiva, nos hallamos frente a un tema complejo, cuya investigación -aún incipiente en el estudio de las relaciones sociales entre sociedad receptora y migrantes- devela cuáles son los modos más contemporáneos (o que reviven viejos debates) de vincular abordajes del campo biológico al análisis de problemáticas sociales.

Finalmente, el análisis de las representaciones sociales como instrumento de interpretación de las conductas sociales, nos permite acercarnos a las percepciones que la sociedad receptora construye, circula y reproduce acerca de los migrantes externos, lo cual colabora en la comprensión de los mecanismos de reproducción de las desigualdades sociales y cómo éstos necesitan siempre reactualizar sus fundamentos.

\section{Reflexiones finales}


Como se desprende del recorrido realizado, el fenómeno de la educación y la diversidad migratoria puede ser abordado desde diversos paradigmas y perspectivas. Sin embargo, aún quedan múltiples aspectos sobre los que profundizar e incluso arriesgar nuevas visiones que vinculen el impacto que en la sociedad receptora tiene la presencia de estudiantes de origen migrante, las estrategias educativas, la reproducción de discursos y representaciones sociales, y los diferentes procesos que tienden a la reproducción de las desigualdades, no sólo educativas. Nuestro interés, en consecuencia, radica en indagar en los aspectos que refieren a la diversidad migratoria y los dispositivos y técnicas de normalización que se re-producen en el ámbito escolar incorporando una dimensión escasamente explorada, aquella que remite a la paulatina y creciente difusión de los avances de neurociencias, en particular, en vínculo con el campo educativo. En tal sentido, los recientes resultados en la investigación neurocientífica expanden su alcance a un punto en el que tales disciplinas están siendo concebidas como aportantes de insumos teóricos para el desarrollo de políticas públicas en educación, así como también en tanto herramientas analíticas para el diseño de estrategias didácticas de aplicación en el aula.

Un aporte teórico fundamental para el planteo de nuestro problema de investigación surge de las ideas acerca del "racismo de la inteligencia" desarrolladas por Bourdieu (s/f: 1) y que se enmarcan en las nuevas formas del racismo que precisan de modos eufemizados de expresarse dada la censura que aquel racismo clásico bio- logicista representa y “... si se invoca el discurso científico para justificar el racismo de la inteligencia, esto no se debe sólo a que la ciencia representa la forma dominante del discurso legítimo, también y sobre todo se debe a que un poder que cree estar fundado en la ciencia, un poder de tipo tecnocrático recurre naturalmente a la ciencia para fundar su poder".

Tal como se señaló, se trata de un proyecto de carácter exploratorio que se encuentra en su fase inicial y que propone una mirada novedosa en torno al vínculo entre los discursos de la neurociencia educativa y las relaciones interculturales en la escuela. Teniendo en cuenta que en los procesos de enseñanza y aprendizaje confluyen numerosas variables (neurales, psicológicas, socioeconómicas, ambientales, entre otras) y que, por lo tanto, dicho proceso de ningún modo puede ser reducido ni explicado por la actividad neural, nos preguntamos: ¿por qué los discursos sobre las neurociencias educativas adquieren tanta difusión y prestigio entre ciertos educadores y gestores de políticas públicas educativas? ¿de qué modo incide el halo de carácter "científico" bajo el cual son presentados sus postulados e ideas a docentes y demás miembros de la comunidad educativa? ¿cómo piensan las neurociencias un aula diversa? ¿tienen las neurociencias una función normalizadora que patologiza la diferencia y la diversidad?

Estos interrogantes nos sitúan ante otro más general: ¿estamos ante un nuevo "racismo de la inteligencia"? 


\section{Referencias citadas:}

Battro, A. M. y Cardinali, D. P. (1996): “Más cerebro en la educación”. Buenos Aires, La nación.

Battro, A. M. (2011): “Neuroeducación: El cerebro en la escuela". En La Pizarra de Babel: Puentes entre neurociencia, psicología y educación. Lipina, S. y Sigman, M. (Editores). Buenos Aires, El Zorzal.

Bauman, Z. (2003): "De la igualdad al multiculturalismo". En Z. Bauman, Comunidad. En busca de seguridad en un mundo hostil. Madrid, Siglo XXI, 107-129

Bertoni, L.A. (2007): “La escuela y la formación de la nacionalidad, 1884-1890". En. Bertoni, L.A. Patriotas, cosmopolitas y nacionalistas. La construcción de la nacionalidad argentina a fines del siglo XIX. Buenos Aires, Fondo de Cultura Económica, pp. 41-77.

Bourdieu, P. (1990): "El racismo de la inteligencia". Intervención en el Coloquio del MRAP en mayo de 1978, publicada en Cahiers Droit et liberté (Races, sociétés et aptitudes: apports et limites de la science), núm. 382, pp. 67-71. Disponible en web:

http://sociologiageneral.sociales.uba.ar/wp-content/uploads/sites/115/2013/06/Pierre-Bourdieu-El-racismo-de-la-inteligencia.pdf [Consulta: 10 de agosto de 2019]

Bowers, J. S. (2016): “The Practical and Principled Problems With Educational neuroscience”, Psychological Review, 4(2), pp. 27-36.

Bruer, J. (1997): “Education and the Brain: A Bridge
Too Far”. Educational Researcher, 26(8), pp. 4-16.

Bruer, J. (2016): “Neuroeducación: Un panorama desde el puente”, Revista Propuesta Educativa, 2(46), pp. 14-25. Disponible en web: http://www.propuestaeducativa.flacso.org.ar/archivos/dossier_articulos/102.pdf [Consulta: 10 de agosto de 2019]

Byrnes, J.P. y Fox, N.A. (1998): “The educational relevance of research in cognitive neuroscience", Educational Psychology Review, 10, pp. 297-342.

Carli, S (2017): La educación pública como laboratorio social: neurociencias y espiritualidades empresariales. Disponible en web: https://conversacionesnecesarias.org/2017/07/03/la-educacion-publica-como-laboratorio-social-neurociencias-y-espiritualidades-empresariales/ [Consulta: 10 de agosto de 2019]

Castorina, J. A. (2016): “La relación problemática entre neurociencia y educación. Condiciones y análisis crítico", Revista Propuesta Educativa, 2(46), pp. 14-25. Disponible en web: http://www.propuestaeducativa.flacso.org.ar/dossier_articulo.php?i$\mathrm{d}=103 \& \mathrm{cnum}=46$ [Consulta: 10 de agosto de 2019]

Cebolla Boado, H. (2007): “La concentración de minorías étnicas en las escuelas británicas: un análisis sobre la elección de centros", Revista Española de Investigaciones Sociológicas, 118, pp: 97-121. Disponible en web: http://www.reis.cis.es/REIS/PDF/ REIS_118_061181906238093.pdf [Consulta: 10 de agosto de 2019] 
Dekker, S., Lee, N., Howard Jones, P. y Jolles, J. (2012): "Neuromyths in Education: Prevalence and Predictors of Misconceptions among Teachers", Frontiers in Psychology, 3(2), pp. 415- 429.

De Vos, J. (2016): “The Educated Brain: A Critique of Neuroeducation”. De Vos, J. (Eds.),The Metamorphoses of the Brain-Neurologisation and its Discontents. Ghent, Palgrave MacMillan.

Devoto, F. (1992): “Movimientos migratorios: historiografía y problemas”. Buenos Aires, Centro Editor de América Latina.

Devoto, F. (2003): “Historia de la inmigración en la Argentina”. Buenos Aires, Sudamericana.

Diez, M y Novaro G. (2011): “ ¿Una inclusión silenciosa o las sutiles formas de la discriminación? Reflexiones a propósito de la escolarización de niños bolivianos". En Courtis, C. y Pacecca, M. I. Discriminaciones étnicas y nacionales. Un diagnóstico participativo. Buenos Aires, Editores del Puerto y Asociación por los Derechos Civiles.

Diez, M., Novaro, G. y Martínez, L. V. (2017): “Distinción, jerarquía e igualdad. Algunas claves para pensar la educación en contextos de migración y pobreza", Cuadernos del Instituto Nacional de Antropología y Pensamiento Latinoamericano, 26(2), pp. 23-40.

Douglas, M. (1996): "Las instituciones se ocupan de la clasificación”. En Douglas M. Cómo piensan las instituciones, Madrid, Alianza Editorial, pp. 135-160
Dubet, F. (2011): “Repensar la justicia social”. Buenos Aires, Siglo XXI.

Duschatzky, S. (1996): “De la diversidad de la escuela a la escuela de la diversidad", Revista Propuesta Educativa, 15.

Duschatzky, S. y Skliar, C. (2000): “La diversidad bajo sospecha. Reflexiones sobre los discursos de la diversidad y sus implicancias educativas", Cuadernos de Pedagogía Rosario, Año IV, $\mathrm{N}^{\circ}$ 7, Centro de Estudios en Pedagogía crítica, Rosario.

Ferreres, A. y Abusamra, V. (2016): "Neurociencias y educación”. Buenos Aires, Paidós.

Finocchio, S. (2009): “Migración y educación: sentidos y desafíos de una trama con matices", Revista Todavía, Pensamiento y cultura en América Latina, 23, pp. 9-13.

Gagliano, F. (1991): Nacionalismo, educación y pluralismo cultural. Polémicas educativas en torno del Centenario. En Puiggrós, A. Sociedad civil y Estado en los orígenes del sistema educativo argentino. Buenos Aires: Galerna.

Jodelet, D. (1986): La representación social: fenómenos, concepto y teoría. En Moscovici, S. Psicología social, Barcelona, Paidós, pp.469-494.

Jodelet, D. y Balduzzi, M. M. (2011): “Aportes del enfoque de las representaciones sociales al campo de la educación", Espacios en blanco, Serie indagaciones, 21(1), pp. 133-154. Disponible en web: http://www. 
redalyc.org/articulo.oa?id=384539803006 [Consulta: 10 de agosto de 2019]

Jordán, J. (1994): “La escuela multicultural”. Buenos Aires: Paidós.

Jordán, J. (2003): “Educar para la convivencia intercultural en sociedades multiculturales", Revista de Educación, Número extraordinario 1, Ministerio de Educación, Cultura y Deporte, Madrid.

Juliano, D. (1993): “Educación intercultural. Escuelas y minorías étnicas”. Madrid, Eudema.

Lipina, S. (2016): “Introducción. Actualizaciones en neurociencia educacional", Revista Propuesta Educativa, 2(46), pp. 6-13. Disponible en web: http://www. propuestaeducativa.flacso.org.ar/dossier_introduccion.php?num=46 [Consulta: 10 de agosto de 2019]

Manes, F. (2013): “Introducción”. En De Podestar, M. E et. al (comp.) El cerebro que aprende. Buenos Aires, Aique.

Marcus, G. (2013): "The Problem with the Neuroscience Backlash", The New Yorker. Disponible en web: http://www.newyorker.com/tech/elements/ the-problem-with-the-neuroscience [Consulta: 10 de agosto de 2019]

McCarthy, C. (1994): "Racismo y curriculum. La desigualdad social y las teorías políticas de las diferencias en la investigación contemporánea sobre la enseñanza”. A Coruña, Fundación Paideia/Ec. Morata.
Merlin, N. (2017): “El feliz mundo neoliberal". Disponible en web: https://www.pagina12.com. ar/41342-el-feliz-mundo-neoliberal [Consulta: $10 \mathrm{de}$ agosto de 2019]

Ministerio de Educación de la Nación (Ed.). (s. f.): "Aprender con el cerebro en mente". Fundación INECO.

Neufeld, M. y Thisted, J. (comps.) (2001): “De eso no se habla... los usos de la diversidad sociocultural en la escuela." Buenos Aires, Eudeba.

OCDE (2007): “La comprensión del cerebro. El nacimiento de una ciencia del aprendizaje". Santiago, Ediciones Universidad Católica Silva Henríquez

Pallarés Domínguez, D. (2016): "Neuroeducación en diálogo: neuromitos en el proceso de enseñanza-aprendizaje y en la educación moral", Pensamiento, 72 (273), pp. 941-958.

Raspall, L (2017): “Neurociencia para educadores". Rosario, Homo Sapiens Ediciones.

Terigi, F. (2016): "Sobre aprendizaje escolar y neurociencias", Revista Propuesta Educativa. (2)46, pp. 50-64. Disponible en web: http://www.propuestaeducativa.flacso.org.ar/dossier_articulo.php?i$\mathrm{d}=105 \&$ num $=46$ [Consulta: 10 de agosto de 2019]

\section{Notas:}

${ }^{1}$ Se trata de una serie de materiales informativos dirigidos a docentes de todos los niveles educativos 
con miras a vincular y articular las neurociencias y la práctica educativa. Este trabajo se realiza en el marco del laboratorio de Neurociencia y Educación que funciona en el Ministerio de Educación, en línea con el Plan Estratégico Nacional "Argentina Enseña y Aprende" 2016-2021. 